\title{
Descriptions and Unknowability
}

\author{
Jan Heylen
}

In a recent paper Horsten embarked on a journey along the limits of the domain of the unknowable. Rather than knowability simpliciter, he considered a priori knowability, and by the latter he meant absolute provability, i.e. provability that is not relativized to a formal system. He presented an argument for the conclusion that it is not absolutely provable that there is a natural number of which it is true but absolutely unprovable that it has a certain property. Informally glossed, Horsten's argument runs as follows:

\begin{abstract}
[1] Suppose, for a reductio, that there exists a property $\theta$ of natural numbers such that it is provable that for some natural number $n, \theta(n)$ is true but unprovable. [2] Then, by the least number principle, there must be a smallest such natural number $n$. [3] Then there provably exists exactly one smallest number $n$ such that $\theta(n)$ is true but unprovable. [4] Then it is provable that the smallest $n$ such that $\theta(n)$ is true but unprovable is true but unprovable. [5] But then $\theta(n)$ is both provable and unprovable. [6] But this is a contradiction. So there can be no property of natural numbers $\theta(n)$ such that it is provable that for some number $n, \theta(n)$ is true but unprovable. $[(?$, p. 1$)$; the numbering is mine $]$
\end{abstract}

It is crucial for the argument to work that the following description principle is correct:

If it is provable that a given property $\phi(x)$ stated in the language of arithmetic plus the concept of knowability is uniquely satisfied, then there is a description term $\iota x \phi(x)$ ('the $\phi$ ') such that $\phi(\iota x \phi(x))$ holds. (?, p. 241)

Because his argument essentially involves the above description principle, Horsten calls his argument the description argument. In what follows I will use the above description principle in an argument for the collapse of provability into truth - a collapse argument, in short. The collapse argument consists of two main steps.

First, I will prove that, for every $\phi$, there is a unique number $n$ that satisfies the following property:

$n$ equals 1 iff $\phi$ is true and $n$ equals 0 iff $\phi$ is false and it is provable that $n$ equals 1 or it is provable that $n$ equals 0

Call this property ' $\phi^{*}$ '. Suppose that $\phi$ is true. Then if one replaces $n$ with 1 , then one gets a theorem, $\phi^{*}(1)$. Suppose that $\phi$ is false. Then if one replaces 
$n$ with 0 , then one gets a theorem, $\phi^{*}(0)$. Either $\phi$ is true, or it is not. So either $\phi^{*}(1)$ is true, or $\phi^{*}(0)$ is. By existential generalisation on both disjuncts, there is a natural number $n$, such that $\phi^{*}(n)$, or there is a natural number $n$, such that $\phi^{*}(n)$. Either way, there is a natural number $n$, such that $\phi^{*}(n)$. It remains to be established that $n$ is unique. Suppose it is not. Then there is another natural number $m$, such that $\phi^{*}(m)$, and $m$ differs from $n$. Now suppose that $\phi$ is true. Then both $n$ and $m$ equal 1 and, therefore, by the transitivity of identity, $n$ equals $m$. Contradiction. Similarly in the case that $\phi$ is false. To conclude, there is a unique natural number $n$, such that $\phi^{*}(n)$.

Second, the above fact leads, in combination with the description principle, to the conclusion that provability collapses into truth. By the closure of provability under theoremhood, it is provable that it is provable that there is a unique natural number $n$, such that $\phi^{*}(n)$. It follows by the description principle and the closure of provability under theoremhood and modus ponens that it is provable that:

$\iota x \phi^{*}(x)$ equals 1 iff $\phi$ is true and $\iota x \phi^{*}(x)$ equals 0 iff $\phi$ is false and it is provable that $\iota x \phi^{*}(x)$ equals 1 or it is provable that $\iota x \phi^{*}(x)$ equals 0

Then it follows by the closure of provability under modus ponens and the factivity of provability that:

it is provable that $\iota x \phi^{*}(x)$ equals 1 iff it is provable that $\phi$ is true and it is provable that $\iota x \phi^{*}(x)$ equals 0 iff it is provable that $\phi$ is false and it is provable that $\iota x \phi^{*}(x)$ equals 1 or it is provable that $\iota x \phi^{*}(x)$ equals 0

It is a tautological consequence that is is either provable that $\phi$ is true, or it is provable that $\phi$ is false. Suppose that $\phi$ is true. Then, by the factivity of provability, it is not the case that it is provable that $\phi$ is false. Hence, it is provable that $\phi$ is true. This concludes the collapse argument.

The collapse argument ought to be accepted by anyone who accepts the description argument. Horsten is committed to a certain theory, call it Horsten's theory, of which all principles are: (a) either explicitly or implicitly used in the description argument, or (b) are sound on his informal interpretation of the vocabulary. I leave it to the reader to verify this claim. Moreover, the conclusion of the collapse argument is relevant for anyone who wants to evaluate the description argument. Let me explain this briefly.

Opinion is divided over the question whether all arithmetical truths are provable or not. Whoever thinks that all arithmetical truths are ultimately provable must accept the description principle if one is willing to allow description terms, for it is simply a theorem of any reasonable first-order logic with description terms. However, the conclusion of the description argument becomes trivial. Indeed, it says nothing more then that there is no property $\phi$ and natural number $n$ such that it both true and false that $\phi(n)$. On the other hand, those who accept that there are unprovable arithmetical truths have to 
reject the conclusion of the collapse argument and, therefore, at least one of its assumptions. To sum up, either all arithmetical truth are provable, or they are not. It follows that either the description argument is sound but its conclusion is trivial, or the description argument is unsound. ${ }^{1}$

\section{K.U. Leuven}

Dekenstraat 2, postbox 03220

3000 Leuven, Belgium

jan.heylen@hiw.kuleuven.be

\section{References}

Horsten, L. (2009). An argument concerning the unknowable. Analysis 69(2), 240-242.

${ }^{1}$ Thanks to Leon Horsten for a fruitful exchange on this subject matter. This work was supported by a grant of the Research Foundation Flanders. 